\title{
Disrupting off-target Cas9 activity in the liver
}

\author{
Off-target genome editing in the liver can be reduced by using lipid nanoparticles to deliver oligonucleotides that \\ disrupt the secondary structure of single-guide RNAs as well as short interfering RNAs targeting Cas9 mRNA.
}

\section{Sean A. Dilliard and Daniel J. Siegwart}

T he COVID-19 vaccines that use lipid nanoparticles (LNPs) to deliver messenger RNA (mRNA) have highlighted the medical and societal benefits of these technologies ${ }^{1}$. mRNA can also be used to induce cells to produce exogenous proteins for genome editing, protein replacement therapy, and immunotherapy. In genome editing (particularly via clustered regularly interspaced short palindromic repeats (CRISPR)), it is crucial to ensure specific on-target editing in diseased cells and to avoid altering the genome of bystander cells ${ }^{2}$. Because a substantial fraction (30-90\%) of an intravenously injected dosage of LNPs accumulates in the liver ${ }^{3}$, unintended (that is, off-target) editing in this organ should be minimized ${ }^{4}$.

There are passive, active and endogenous targeting methods to increase the uptake of nanoparticles by specific tissues and cells. In particular, targeting ligands can be conjugated to the surface of LNPs to enhance binding and endocytosis by specific cellular populations ${ }^{5,6}$ (active targeting), and the composition of LNPs can be adjusted to favour the binding of distinct proteins in the blood that can direct the LNPs to specific organs $^{7,8}$ (endogenous targeting). Off-target editing can also be minimized by rationally selecting a target gene with tissue-restricted expression or disease-specific mutations ${ }^{9}$. In addition, 'anti-CRISPR' molecules can be used to interfere with the activity of CRISPR-associated (Cas) genome-editing proteins $^{10,11}$. Reporting in Nature Biomedical Engineering, James Dahlman and colleagues now show that LNPs can be used to preferentially deliver two types of anti-CRISPR nucleic acid to the liver to disrupt genome editing in hepatocytes, thereby improving the specificity of CRISPR-Cas genome editing in extrahepatic tissues and organs ${ }^{12}$.

Dahlman and co-authors first developed inhibitory anti-CRISPR oligonucleotides that interact with sequence-complementary single-guide RNA (sgRNA) to reduce genome-editing activity mediated by CRISPR-associated protein 9 (Cas9), which they show via the quantification of

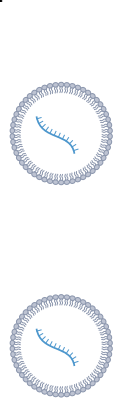

b

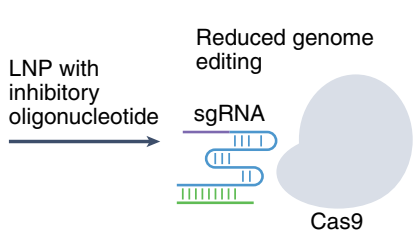

LNP with Genome editing

control

oligonucleotide

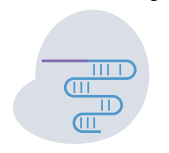

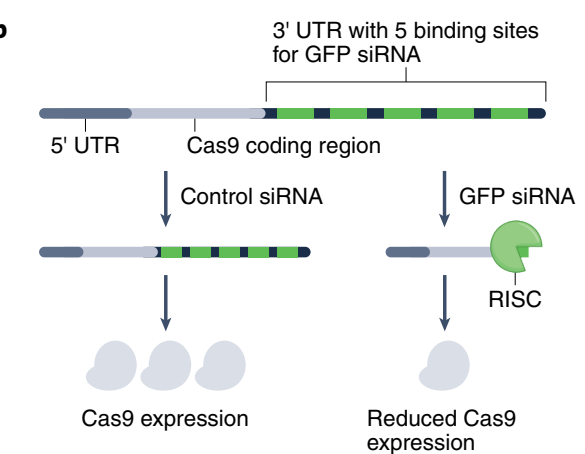

expression
C

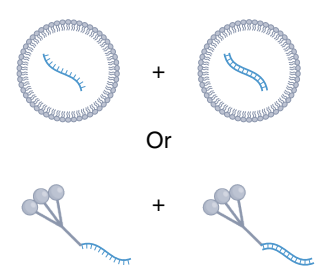

Delivery of liver-targeting inhibitory molecules

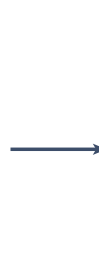

Delivery of the genome-editing machinery

LNP Cas9

$$
\text { mRNA }
$$$$
\text { sgRNA }
$$

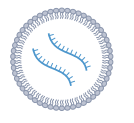

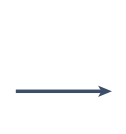

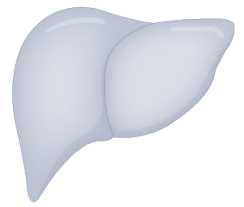

Reduced off-target editing in the liver

Fig. 1 | Inhibitory oligonucleotides to reduce off-target genome editing in the liver. a, Anti-CRISPR oligonucleotides can disrupt the secondary structure of sgRNAs via Watson-Crick base pairing, preventing sgRNAs from binding to Cas9, thereby impeding genome editing. b, Cas9 mRNA can be programmed to be degradable by siRNA, impairing Cas 9 production and thus genome editing. UTR, untranslated region; RISC, RNA-induced silencing complex. c, The simultaneous delivery of anti-CRISPR oligonucleotides and siRNA via LNPs that target hepatocytes (top left) or via GalNAc conjugates (bottom left), followed by the delivery of Cas 9 mRNA and sgRNA via LNPs (middle) that target cells in other organs (such as the lung and spleen) in addition to hepatocytes, reduces genome editing in the liver, thereby improving the tissue specificity of genome editing in the extrahepatic organs. Figure adapted with permission from ref. ${ }^{12}$, Springer Nature Ltd.

insertion-deletion mutations (indels) in immortalized mouse aortic endothelial cells either stably or transiently expressing Cas9. Additional in vitro studies allowed the authors to elucidate the mechanism of action of the anti-CRISPR oligonucleotides. In an in situ DNA-cutting assay, sufficiently high doses of the oligonucleotides reduced DNA cleavage when pre-complexed with sgRNA, but failed to reduce DNA cleavage when incubated with a pre-formed sgRNACas9 complex or when competing with its formation. Also, truncated variants of the anti-CRISPR oligonucleotides that do not bind to the polyU region of the sgRNA did not prevent Cas9 genome editing. The authors concluded that the anti-CRISPR oligonucleotides disrupt the secondary structure of the sgRNA, thereby preventing sgRNA-Cas9 complexation and, hence, the editing of the genome (Fig. 1a).

To investigate whether anti-CRISPR oligonucleotides could be used to reduce off-target editing in the liver, Dahlman and co-authors first treated mice stably expressing Cas 9 tagged with green fluorescence protein (GFP) with either anti-CRISPR oligonucleotides or scrambled oligonucleotides formulated in LNPs (composed of the ionizable lipid cKK-E12, 
the phospholipid 1,2-distearoyl-sn-glycero3-phosphocholine, cholesterol, and the poly(ethylene glycol)-functionalized lipid $\mathrm{C}_{14} \mathrm{PEG}_{2000}$ ) that preferentially target the liver. Two hours later, they treated the same mice with the same LNPs, yet instead encapsulated sgRNA targeting the GFP locus. Compared with the scrambled oligonucleotides, the anti-CRISPR oligonucleotides reduced the frequency of indels in hepatocytes by more than twofold. The authors then show that when pre-treating the mice with either the anti-CRISPR oligonucleotides or the scrambled control oligonucleotides formulated in the same liver-targeting LNPs two hours before injecting the mice with sgGFP-loaded LNPs (composed of the lipid oligomer $7 \mathrm{Cl}$ and $\mathrm{C}_{14} \mathrm{PEG}_{2000}$ ) targeting lung endothelial cells and liver cells, the anti-CRISPR oligonucleotides reduced the frequency of indels in hepatocytes but not in lung endothelial cells. Hence, by using LNPs that target the liver and other organs in combination with hepatocyte-targeting LNPs that contain anti-CRISPR

oligonucleotides, on-target Cas9-mediated genome editing in extrahepatic tissues can be maintained while limiting off-target editing in the liver.

To further reduce off-target genome editing, Dahlman and co-authors designed a Cas9 mRNA incorporating (in its 3' untranslated region) five binding sites for a short interfering RNA (siRNA) specific for GFP (Fig. 1b). In immortalized mouse aortic endothelial cells, the binding of the siRNA to the Cas 9 mRNA activated the endogenous cellular process of RNA interference and resulted in mRNA degradation, leading to reduced Cas9 expression and impaired genome-editing efficacy. In mice, the intravenous injection of siRNA encapsulated in hepatocyte-targeting LNPs 14 hours before an injection of the same LNPs loaded with Cas9 mRNA and a sgRNA targeting the intercellular adhesion molecule 2 resulted in a lower fraction of indels. Similarly, the use of LNPs (composed of 7C1, 1,2-dioleoyl-sn-glycero- 3-phosphoethanolamine, cholesterol, and $\mathrm{C}_{14} \mathrm{PEG}_{2000}$ ) targeting both splenic endothelial cells and hepatocytes after pre-treatment with siRNA formulated in hepatocyte-targeting LNPs led to lower frequencies of indels in the hepatocytes of the mice. The combination of anti-CRISPR oligonucleotides and siRNA further decreased the off-target genome editing of hepatocytes (Fig. 1c). The authors replicated these findings with another delivery system (trivalent $\mathrm{N}$-acetylgalactosamine (GalNAc) conjugates) and in a mouse model of lung inflammation.

The use of anti-CRISPR molecules to reduce genome-editing activity in the liver did not require the development of new LNP formulations for the targeted delivery of the CRISPR-Cas machinery to extrahepatic tissues; rather, off-target liver editing could generally be reduced through the deployment of anti-CRISPR oligonucleotides via LNPs or GalNAc conjugates. It is thus reasonable to expect that the effects of anti-CRISPR molecules could be further enhanced by LNPs designed to specifically target extrahepatic cells and organs $s^{4,7}$ or via the delivery of mRNA transcripts that are degraded by microRNAs endogenously expressed in off-target cells $s^{13}$. Leveraging the unique strengths of anti-CRISPR molecules, designer LNPs and degradation-prone mRNA transcripts could be used to generate disease models, and for therapeutic purposes. However, the multiple components of the system and the currently limited potency of anti-CRISPR oligonucleotides limit the translational prospects of the approach for therapeutic use.

Most contemporary genome-editing technologies (in particular, CRISPR-Cas9, base editing and prime editing) require multiple components (for traditional Cas9-mediated editing, an expression system for the genome-editing protein, a sgRNA and, for certain uses, a template $\mathrm{DNA}^{2}$ ). Hence, the optimization of the design of LNPs for the sequential or simultaneous delivery of each component is essential to make therapeutic in vivo genome editing safer for use in humans ${ }^{14}$. The design of specific delivery systems for genome editing will also aid the development of cell and animal models of disease, drug discovery, and basic science work ${ }^{4}$. Beyond genome editing, the delivery of multiple nucleic acids could be employed to overcome key delivery barriers in cancer and other diseases to improve the efficacy of therapeutics ${ }^{15}$. Moreover, LNPs that preferentially access specific organs and cell types via active or endogenous targeting and that can deliver multiple nucleic acids are likely to facilitate the application of gene editing in new disease settings ${ }^{4}$.

Sean A. Dilliard (D) and Daniel J. Siegwart (D) $₫$ Department of Biochemistry, Simmons Comprehensive Cancer Center, The University of Texas Southwestern Medical Center, Dallas, TX, USA.

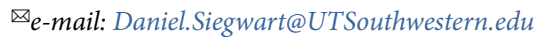

Published online: 21 February 2022

https://doi.org/10.1038/s41551-022-00848-8

References

1. Chaudhary, N., Weissman, D. \& Whitehead, K. A. Nat. Rev. Drug Discov. 20, 817-838 (2021).

2. Doudna, J. A. Nature 578, 229-236 (2020).

3. Zhang, Y.-N. et al. J. Control. Release 240, 332-348 (2016).

Wei, T. et al. ACS Nano 14, 9243-9262 (2020).

5. Veiga, N., Diesendruck, Y. \& Peer, D. Adv. Drug Deliver. Rev. 159, 364-376 (2020).

6. Rurik Joel, G. et al. Science 375, 91-96 (2022).

7. Cheng, Q. et al. Nat. Nanotechnol. 15, 313-320 (2020).

8. Dilliard, S. A., Cheng, Q. \& Siegwart, D. J. Proc. Natl Acad. Sci. USA 118, e2109256118 (2021)

9. Gillmore, J. D. et al. N. Engl. J. Med. 385, 493-502 (2021)

10. Pawluk, A., Davidson, A. R. \& Maxwell, K. L. Nat. Rev. Microbiol. 16, 12-17 (2018).

11. Brown, W., Zhou, W. \& Deiters, A. ChemBioChem 22, 63-72 (2021).

12. Sago, C. D. et al. Nat. Biomed. Eng. https://doi.org/10.1038/ s41551-022-00847-9 (2022).

13. Jain, R. et al. Nucleic Acid Ther. 28, 285-296 (2018).

14. Farbiak, L. et al. Adv. Mater. 33, e2006619 (2021).

15. de Lazaro, I. \& Mooney, D. J. Nat. Mater. 20, 1469-1479 (2021).

\section{Competing interests}

D.J.S. is a co-founder and consultant of ReCode Therapeutics, which has licensed intellectual property from UT Southwestern. S.A.D. and D.J.S. are listed as coinventors on patent applications related to nanoparticle delivery that are filed and owned by the Reagents of the University of Texas System. 